\title{
DIPE: Determination of Input Parameters Uncertainties Methodology Applied to CATHARE V2.5 1*
}

\author{
Jérôme JOUCLA** and Pierre PROBST** \\ ** Institute for Radiological Protection and Nuclear Safety, \\ B.P 17, 92262 Fontenay aux Roses, France \\ E-mail : jerome.joucla@irsn.fr, pierre.probst@irsn.fr
}

\begin{abstract}
Since the revision of the 10 CFR50.46 in 1988, the best-estimate codes may be used in safety demonstration and licensing, provided that uncertainties are added to the relevant output parameters before comparing them with the acceptance criteria. The uncertainty of output parameters comes principally from the lack of knowledge of the input parameters (initial and boundary conditions of the calculated transient, empirical models of the code, etc.). The application of the best estimate plus uncertainty analysis can be made in three steps in a statistical evaluation:

1. The determination of the input parameters (IP) statistical characteristics.

2. The modelling and understanding of the output parameters.

3. The evaluation of the $95^{\text {th }}$ percentile with a high degree of confidence.

The first step is generally done manually according to expert judgments and comparing experimental data. The user effect is also very important in the determination of the statistical characteristics: range of variation and probability law. To reduce this user effect and to help the experts in their evaluation, IRSN has been developing a fully automated methodology and approach. This methodology is called DIPE: Determination of Input Parameters uncertaintiEs. This paper presents the advantages and limits of the application of DIPE for the physical models input parameters of CATHARE V2.5_1, a thermal-hydraulics code used in safety demonstration. DIPE will be applied to separate effect tests data used in the code qualification such as CANON and MARVIKEN.
\end{abstract}

Key words: Statistical Treatment, Nuclear Reactor, Probabilistic Method, Computer Aided Analysis

\section{Introduction}

Paragraph 50.46(a)(1) of 10 CFR part 50 requires that "the uncertainty in the realistic [i.e. best-estimate] evaluation model be quantified and considered when comparing the results of the calculations with the applicable limits in paragraph 50.46(b) so that there is a high probability that the criteria will not be exceeded." In Regulatory Guide 1.157, this "high probability" is expressed as a "95\% or more" probability not to exceed these limits.

In the safety analysis of the large break loss of coolant accident (LB-LOCA), for a best-estimate analysis, the ECCS performance are assessed against the following quantitative criteria: Peak Cladding Temperature (PCT) less than $1204^{\circ} \mathrm{C}$ or $2200^{\circ} \mathrm{F}$, 
maximum Local Clad Oxidation (LCO) less than 17\% and Core Wide Oxidation (CWO) less than $1 \%$. It was proposed that these acceptance criteria might not be exceeded by the $95^{\text {th }}$ percentile estimated with a high degree of confidence (e.g. 95\%). Best-estimate plus uncertainty (BEPU) analysis of the LB-LOCA allows significant margin gains. Therefore the nuclear industry develops and uses widely this type of analysis for power uprating, longer fuel cycles, fuel design etc.

\section{Brief Description of the Method}

IRSN method can be roughly divided in three dependant sequential steps. The end status of each step determines if the method can be followed or not.

The first step of the method deals with the uncertain parameters identification. All the parameters involved in the model correlations are reviewed. The potentially significant ones, regarding the physical phenomenon observed during the target transient, are identified.

During the second step, the basic uncertainties quantification is determined which supposes to be the most difficult one. All of the significant ones have an influence on the uncertainty of the results and have to be evaluated. The quantification method determines the range of uncertain code parameter values obtained from the validation of the code against separate effect tests and for real parameters by using physical data if they exist. These quantifications result in an uncertainty band and a probability density function, the later given generally by experts for all the uncertain parameters.

Third step combines all the basic uncertainty to derive the global uncertainties attached to the expected results. The involved methodology is a fully probabilistic one. The principle is to model each uncertain parameter by a random variable and takes into account the state of knowledge on the parameter through an interval span and a distribution function. Then all the modelled uncertainties are derived with a statistical method to give the whole response uncertainty associated with the computational process used to evaluate the response.

The present paper will deal with the second step as stated above, describing an automated methodology applied on the separate effect tests CANON and MARVIKEN used in the qualification of the CATHARE 2 CODE V2.5_1.

\section{Sources of Uncertainties}

\subsection{Identification of Input Uncertainties}

The goal of the present step is to identify all the sources of uncertainties involved in the calculation. These uncertainties can be classified into three different categories:

1. The uncertainties due to the input data: These can be linked to the unknown experiment or reactor state (e.g. initial and boundary conditions), the unknown physical properties of the materials or the geometrical data. The scenario of the accident can also be a source of uncertainty (e.g. times of events) and fall into this category. These uncertainties are more or less easily available. Usually, experimenters perform uncertainties evaluation on the facilities and therefore provide good material information. 
2. The uncertainties due to the code implementation (i.e. user effect) are probably the most difficult to estimate and to quantify.

3. The uncertainties due to the code conception: These are introduced by the choice of the basic models (e.g. number of fluids, number of fields, and number of equations), the correlations or models (i.e. closure equations), the number of dimensions (0D to $2 \mathrm{D}$ ), the choice of the numerical methods and nodalization. These uncertainties reflect the necessary simplification of the physical reality with respect to the computational cost. It seems obvious that taking into account uncertain parameters related to the number of fluids, the number of fields, the number of equations or the number of dimension would be fairly complex.

Reminding our goal, the previous step has established the list of the models involved during the computation. The uncertain parameters will be deduced from these models. In every correlation, where a coefficient has been experimentally fitted, we will suspect a source of uncertainty and model this coefficient by a random parameter. This paper presents an automated methodology called DIPE to quantify these kinds of input uncertainties.

\subsection{CATHARE2 V2.5_1 mod3.1 Code}

The V2.5_1 mod3.1 version of the CATHARE ${ }^{(1)}$ code (Code for Analysis of Thermal-Hydraulics during an Accident of Reactor and safety Evaluation) is the outcome of more than 20 years of joint development effort by CEA, IRSN, EDF and FRAMATOME. CATHARE is a system code devoted to thermal-hydraulics transient calculations for nuclear reactor safety analysis. Two-phase flows are described using two-fluid six-equation model and the presence of non-condensable gases can be taken into account by one to four additive transport equations. The code allows a three-dimensional modelling of the Pressure Vessel. It comes with a complete physical assessment.

\subsection{Sources of Knowledge}

In the case we try to reproduce or simulate an experimental transient, the whole documentation of the experimental facility is obviously required. Experimental results and reports are also a major part of the source of knowledge. These data are imperative to extract input data uncertainties. Then, the code qualification database is a fundamental basis to quantify basic parameters uncertainties due to the code conception. But among all the separate and integral tests used to validate the code, we have to select those that interest our goal.

The models inventory and documentation and the previously identified parameters of interest prepare the basic frame work. The tests which will be given a full consideration are those that were used to qualify the model's correlations and consequently, to fit the uncertain coefficient.

For example, the separate effects test facility of the VERTICAL CANON will be used because it is very suitable for qualifying the correlations involving momentum \& heat exchange between phases. As well, the PERICLES, MARVIKEN, CREARE and OMEGA experiments will be used for their representativeness of respectively the interfacial friction, the liquid to interface heat transfer, the flooding limit in a complex geometry, the flow stagnation during the blowdown of a LBLOCA. Finally, we always have the expert judgment for the case of lack of knowledge and ambiguity. 


\section{DIPE: Determination of Input Parameters uncertaintiEs}

\subsection{Why DIPE?}

IRSN wished to develop a systematic and a mathematical methodology to estimate the range and the probability law associated to each input uncertain parameters. Indeed, we would like to minimize the expert judgment used to determine the parameters quoted previously because the method used before DIPE was practically based on the expert judgment ${ }^{(2)-(3)-(4)}$.

\subsection{The Principle}

The principle of this method is represented by the algorithm of the figure 1 . The description of the algorithm is illustrated by a theoretical separate effect test using only:

- One input uncertain parameter noted X,

- One output parameter of interest as a pressure.

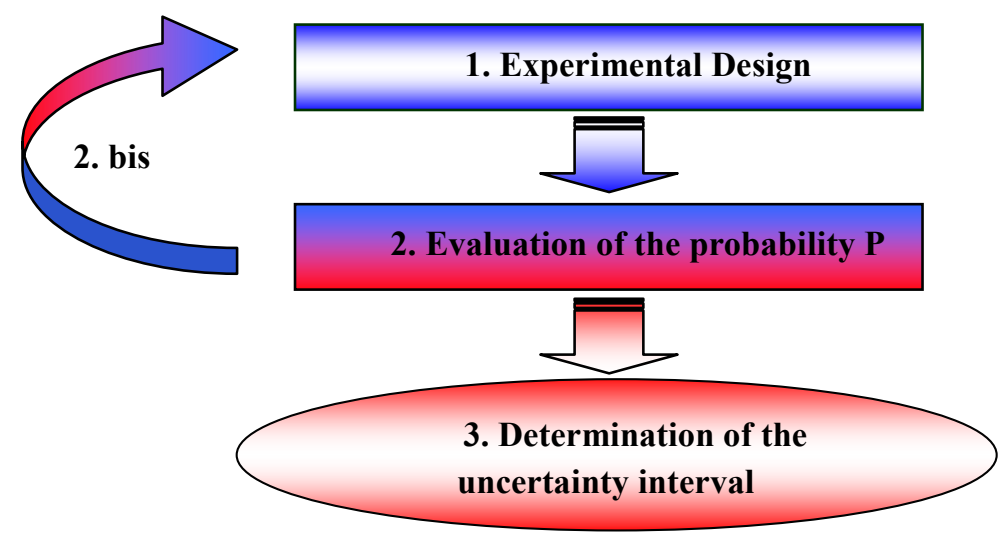

Fig. 1 Principle of DIPE

First Step "Experimental Design": Several simulations are carried out by varying the parameter $\mathrm{X}$ on an interval chosen, and the curves noted $\mathrm{C}(\mathrm{X})$ " calculated by CATHARE are compared with the experimental data noted Cexp, as showed on the figure 2.

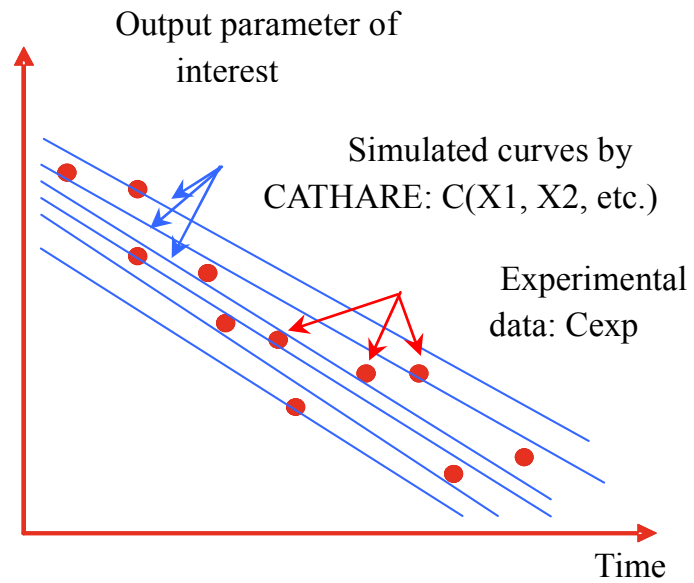

Fig. 2 Experimental curve framed by simulated curves 
Second step “Evaluation of the probability P": For each value of the uncertain parameter noted $\mathrm{Xi}$ (thus for each simulated curve calculated with $\mathrm{Xi}$ noted $\mathrm{C}(\mathrm{Xi})$ ), the ratio, noted $\mathrm{P}(\mathrm{Xi})$, of the number of experimental points (circled on the figure 3 ) above the corresponding simulated curve $\mathrm{C}(\mathrm{Xi})$ and the total number of experimental is calculated, as indicated on figure 3.

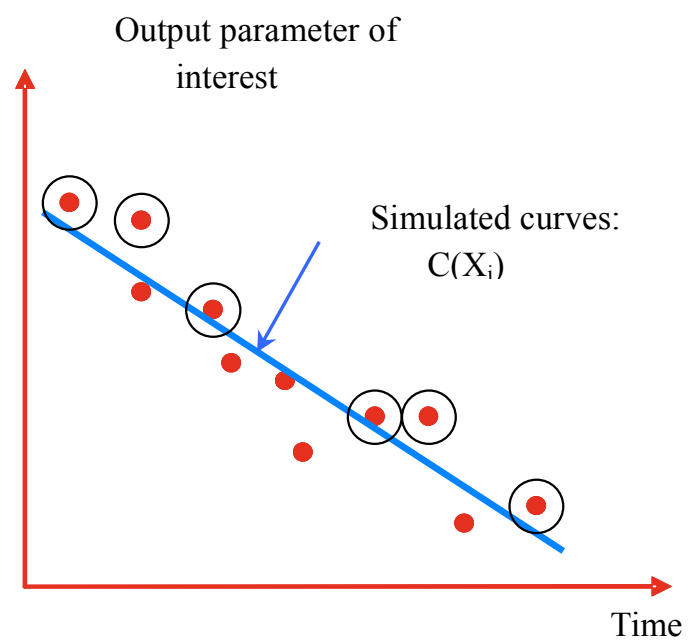

Fig. 3 Calculation of the ratio $\mathrm{P}(\mathrm{Xi})$

We can consider that $\mathrm{P}(\mathrm{Xi})$ is the probability that the simulated curve is lower than the experimental curve. Thus, the function $\mathrm{P}(\mathrm{Xi})$ obtained can be considered as a cumulative density function (CDF). Thus, we can represent it versus the value of the input parameter (see figure 4).

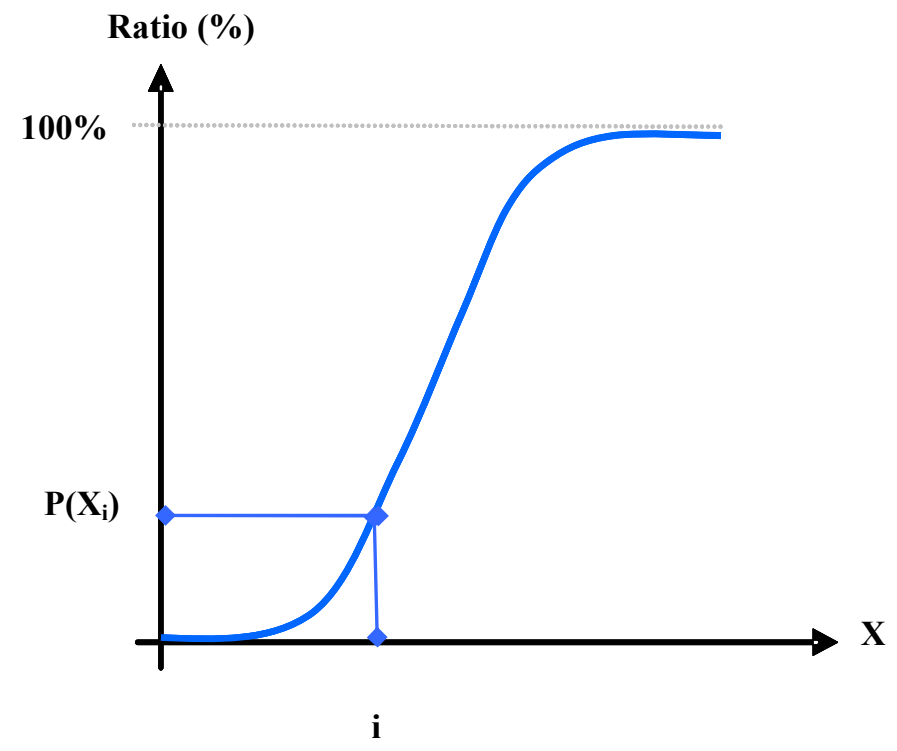

Fig. 4 Cumulative density function built with the ratio $\mathrm{P}(\mathrm{X})$

Second Step "Bis": If the experimental data are not bounded by the entire simulated curves $\mathrm{C}(\mathrm{X})$, we try to enlarge the experimental design to do it.

Third step "Determination of the uncertainty interval": We seek to bound the experimental data with an accuracy of $95 \%$. It corresponds to search the curves $\mathrm{C}(\mathrm{X})$ having 
$2.5 \%$ of the experimental data above and another one having $2.5 \%$ of the experimental data below (see figure 5).

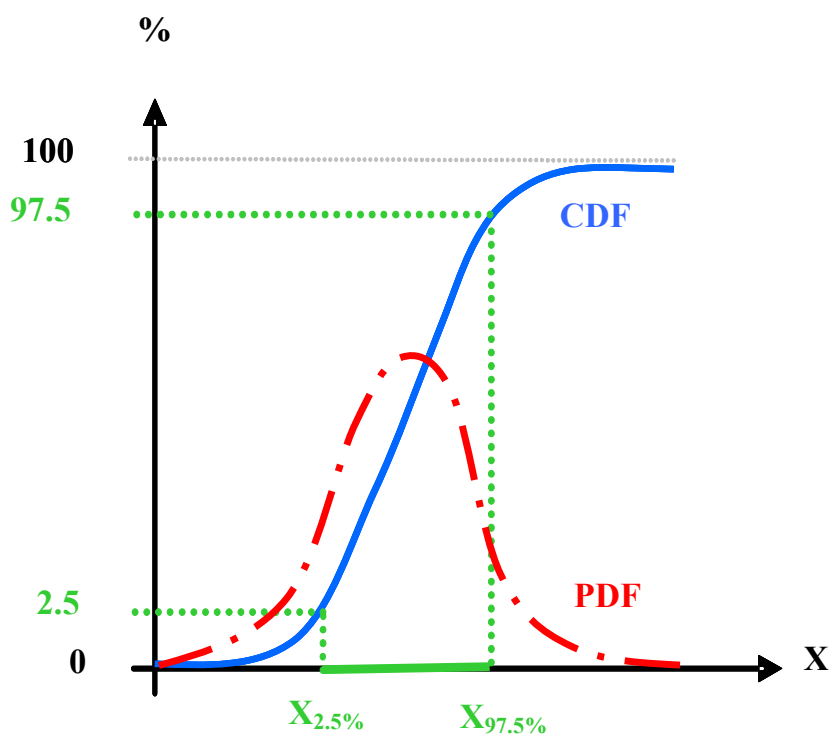

Fig. 5 Determination of the range of the input parameter $\mathrm{X}$

Then we seek the values of $\mathrm{X}$ for which $\mathrm{P}(\mathrm{X})$ takes values 0.025 and 0.975 , i.e. the 2.5 th and the 97.5 th percentile.

Thus, we get the probability density function (PDF) by deriving $\mathrm{P}(\mathrm{X})$.

\subsection{Mathematical Assumptions}

Mathematically, we can justify DIPE with the following assumptions:

- Assumption 1: "Absence of measurement error"

The measurement error is negligible

- Assumption 2: "Random Error of CATHARE"

At each time, it exists a calculated curve $\mathrm{C}(\mathrm{Xi})$ where the uncertain input parameter $\mathrm{Xi}$ is a random variable coming from an independent probability law

- Assumption 3: "Monotony"

At each time, the calculated curve $\mathrm{C}(\mathrm{Xi})$ is monotonous.

We will discuss of these assumptions during the analysis of the application in section 6 .

\section{DIPE Application}

DIPE has been applied to the 46 experiments used in the CATHARE code qualification. Only two are presented in the paper: CANON VERTICAL which uses one uncertain input parameter and MARVIKEN which uses two uncertain input parameters.

\subsection{CANON VERTICAL (CNV)}

CANON VERTICAL ${ }^{(5)}$ (1982, CEA, Grenoble, France) experiment has been carried out to study transient two phase vertical flow with entrainment of liquid. This test facility allows a vertical tube to be depressurized by a small breach at its top. Before each blowdown subcooled water is at rest in the tube at a temperature $\mathrm{T}=230^{\circ} \mathrm{C}$ to $320^{\circ} \mathrm{C}$ and a 
pressure $\mathrm{P}=150$ bar. These experiments make it possible to assess the interfacial friction in slug flow for a tubular geometry. One of the main parameter is the interfacial friction. The inter-facial friction influence on the blowdown is principally visible through the study of the void fractions and the mass inventory in the tube (which are measured in the tests). Because the measurement of the void fraction is very oscillating, we prefer to use only the mass inventory output parameter for the DIPE application.

Table 1 Boundary and initial condition of the analysed CANON VERTICAL tests

\begin{tabular}{|c|c|c|}
\hline $\begin{array}{c}\text { Number of } \\
\text { the experiment }\end{array}$ & $\begin{array}{c}\text { Initial } \\
\text { temperature of } \\
\text { the water }\left({ }^{\circ} \mathrm{C}\right)\end{array}$ & $\begin{array}{c}\text { Diameter } \\
\text { of the Breach } \\
(\mathrm{mm})\end{array}$ \\
\hline 9 & 300 & 3 \\
\hline 22 & 230 & 5 \\
\hline 23 & 320 & 5 \\
\hline 27 & 300 & 10 \\
\hline
\end{tabular}

In the paper, we present the application of DIPE to CNV9, CNV22, CNV23 and CNV27 (see table 1) used in the CATHARE qualification. The input parameter selected is the interfacial friction (churn-bubble flows) in pipe geometry (TOBT1) and the output parameter is the mass inventory in the vertical pipe.

\subsection{MARVIKEN}

The CFT (Critical Flow Test) ${ }^{(6)}$ program was initiated to collect data using different test nozzles representative of pipe sizes in a typical nuclear reactor. The objective of the program was to collect critical flow data as a function of nozzle diameter and length for subcooled and low quality saturated water. One of these kinds of tests was MARVIKEN tests. The tests were conducted by filling the Marviken pressure vessel with water. After heating the vessel water to a specified temperature and pressure level, rupture discs were failed. The vessel contents were discharged through a specific test nozzle into containment. The containment pressure was relieved by discharging a steam-water mixture to the atmosphere, through exhaust pipes. A good modeling of liquid interface heat flux is especially important for a correct prediction of critical flow rate for subcooled or saturated inlet conditions.

The input parameters selected (considered to be influential) are the liquid-interface heat flux - flashing (QLF1) and the liquid-wall friction coefficient in pipe geometry (CL1). The output parameter is the critical flow rate. Two MARVIKEN experiments, MRK17 and MRK24 ${ }^{(7)-(8)}$ (see table 2) are studied.

Table 2 Boundary and initial condition of the analysed MERVIKEN tests

\begin{tabular}{|c|c|c|}
\hline Experiment $\mathrm{N}^{\circ}$ & Diameter of the nozzle $(\mathrm{m})$ & Length of the pipe $(\mathrm{m})$ \\
\hline MRK17 & 0.3 & 1.116 \\
\hline MRK24 & 0.5 & 0.166 \\
\hline
\end{tabular}

\subsection{Running DIPE}

The realization of the first step of DIPE is done with the SUNSET code coupled with 
the CATHARE code. SUNSET means "Statistical UNcertainty and SEnsitivity Tools" and has been developing by IRSN. It was used for generating samples (complete, factorial, HGLG, etc.) but it is also capable of statistical analysis too. The experimental designs used in our study are often complete experimental designs. After getting back the CATHARE transients, the input uncertainties are evaluated by the DIPE toolbox programmed under Matlab language.

\section{Results and Limits of Dipe}

\subsection{CANON VERTICAL: 1 Uncertain Input Parameter}

Four experiments CNV are exploited: CNV9, CNV22, CNV23 and CNV27. You can see an example of framed data on the figure 6 for CNV22.

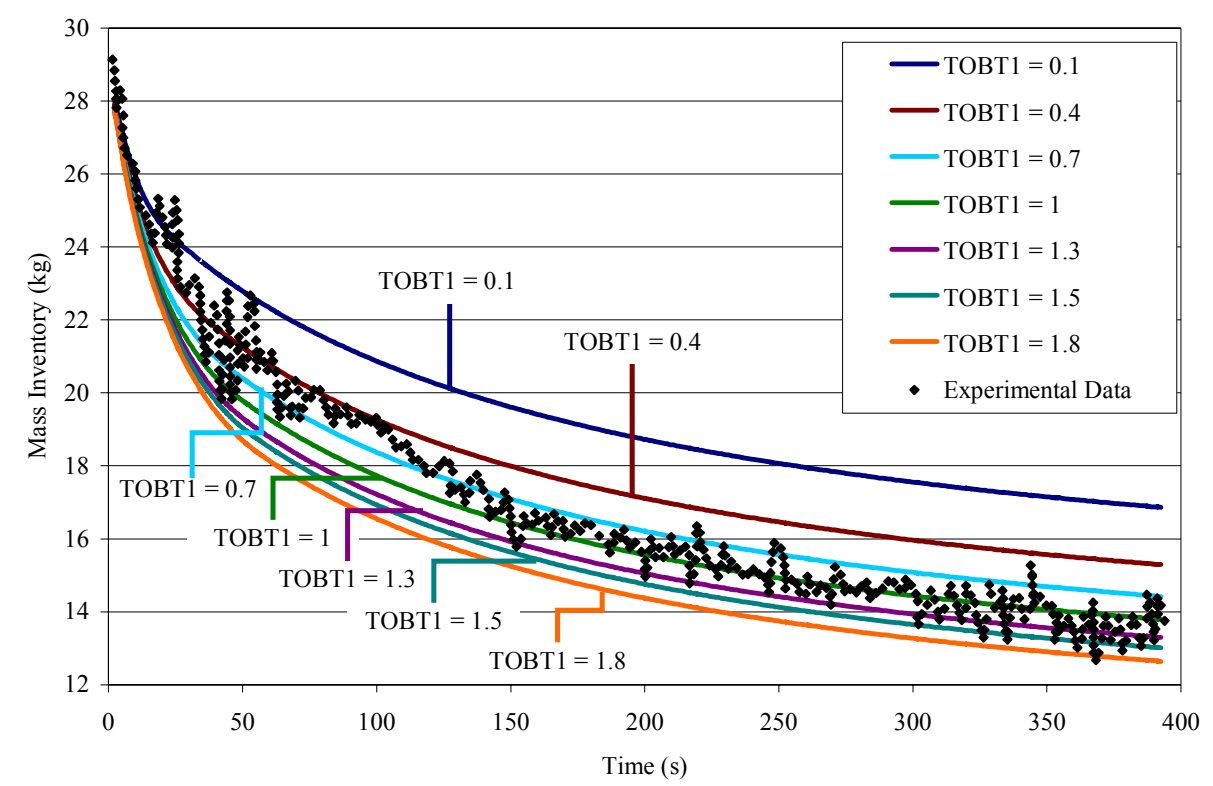

Fig. 6 Experimental data framed by simulated curves for CNV22

They are successively studied in order to compare their probability density functions and their ranges of uncertainty obtained for each one of them.

After using DIPE, we obtained the results shown in the figure 7 to 10 and the table 3 .

Table 3 Range of variation of the TOBT 1

\begin{tabular}{|c|c|c|}
\hline \multirow{2}{*}{} & \multicolumn{2}{|c|}{ TOBT1 } \\
\cline { 2 - 3 } & Inf. & Sup. \\
\hline CNV09 & 2.10 & 12.54 \\
\hline CNV22 & 0.07 & 1.46 \\
\hline CNV23 & 4.08 & 13.87 \\
\hline CNV27 & 0.35 & 1.83 \\
\hline
\end{tabular}

The ranges of variation and the CDF/PDF of the TOBT1 uncertainty are very different according to the experiments which represent different physical conditions in accident studied in the safety analysis. 


\section{Range of Variation}

To apply these results to a real Nuclear Power Plant (NPP), we cannot select only one set of range of variation plus a PDF in safety analysis because it is not representative for all the conditions.

\section{Probability Density Function}

Secondly, it is totally unjustified to suppose a shape to the PDF associated to the parameter TOBT1 to evaluate the input uncertainty. It is not justified to perform a convolution of the four PDFs to create a new one because we do not have all the possible experimental conditions.

\section{To Sum Up}

In order to perform a Best Estimate analysis, for selecting the TOBT1 range of variation, we can take the minimum and maximum values of the found intervals, i.e. 0.07 and 13.87. Finally we select 0.1 and 14 because DIPE is a numerical methodology and 0.07 or 13.87 do not seem to be very adequate to our physical sense.

To select the PDF, as we cannot take a precise PDF, we can select a histogram or a uniform by parts. The uniform by parts distribution is chosen in order to have the reference value 1 of the parameter being the 50th percentile of the PDF law which, on our point of view represents the best estimate character of the code. It will be noted that TOBT1 is involved in other separate effect tests: therefore for determining its uncertainty, we have to take into account all these experiments.

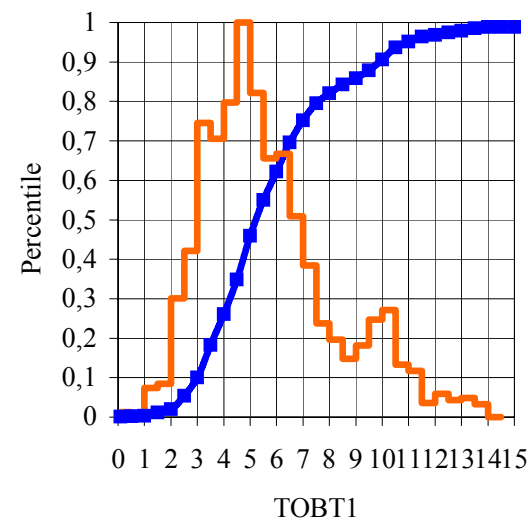

Fig. 7 PDF/CDF - CNV09

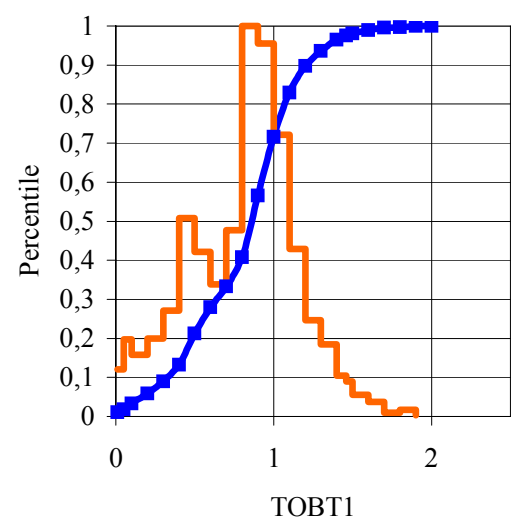

Fig. 9 PDF/CDF - CNV22

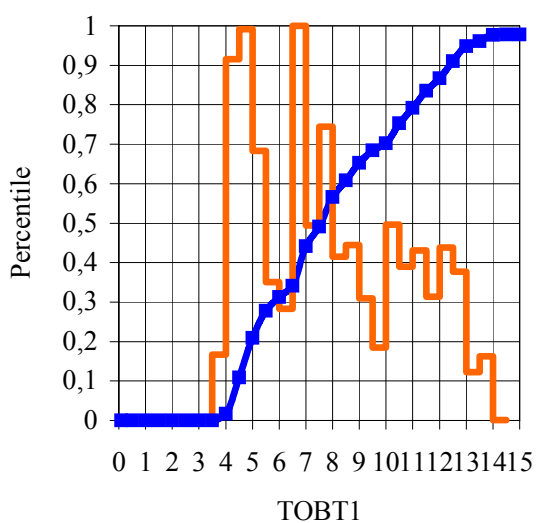

Fig. 8 PDF/CDF - CNV23

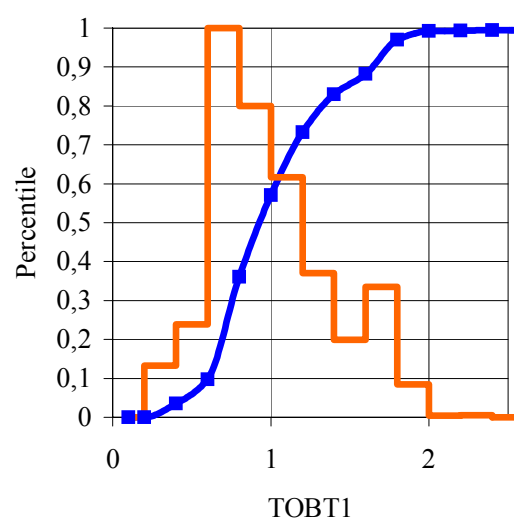

Fig. $10 \mathrm{PDF} / \mathrm{CDF}-\mathrm{CNV} 27$ 


\subsection{MARVIKEN: 2 Uncertain Input Parameters}

DIPE has been applied to the MARVIKEN experiments: MRK17 and MRK24. For each experiment, the three percentiles, i.e. $2.5^{\text {th }}, 50^{\text {th }}$ and $95^{\text {th }}$, are plotted according to the value of the two parameters CL1 and QLF1.

\section{MRK24}

It seems that CL1 has only a small impact on the determination of the range of variation of QLF1, see figure 11. Finally, we can select the limits of the uncertainty intervals of only QLF1: 0.1 and 1 taking the maximum and minimum possible value.

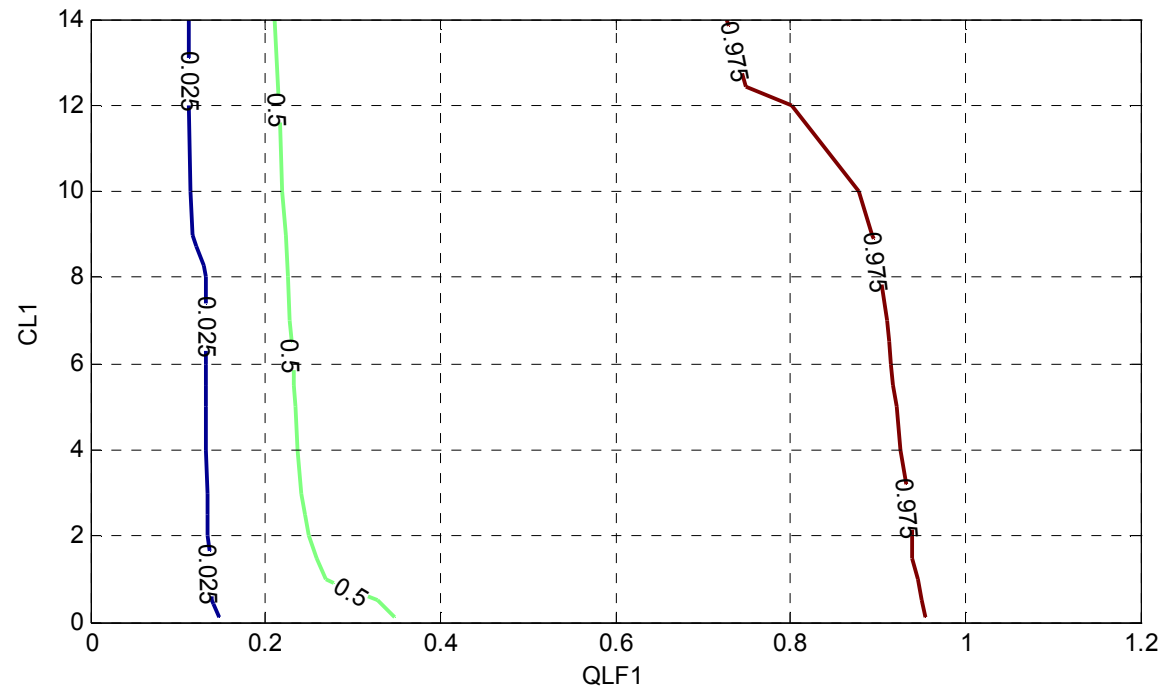

Fig. 11 Evolution of the percentiles according to CL1 and QLF1, MRK24

\section{$\underline{M R K 17}$}

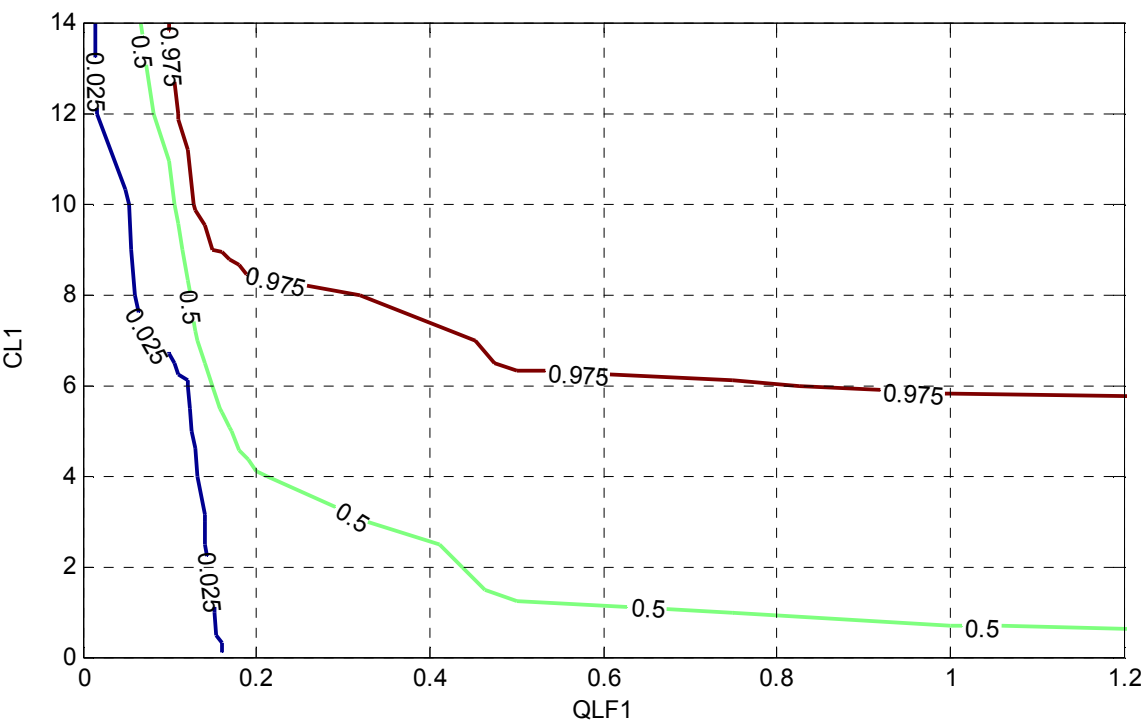

Fig. 12 Evolution of the percentiles according to CL1 and QLF1, MRK17

This experiment is more difficult to analyze because CL1 and QLF1 have an effect on the determination of the uncertainty range as we can see on the figure 12 . 


\section{Dependence}

In the case of this experiment, dependences between uncertainties of the two parameters can be taken into account. The colored area of figure 13 represents the allowed surface for (QLF1, CL1) uncertainties.

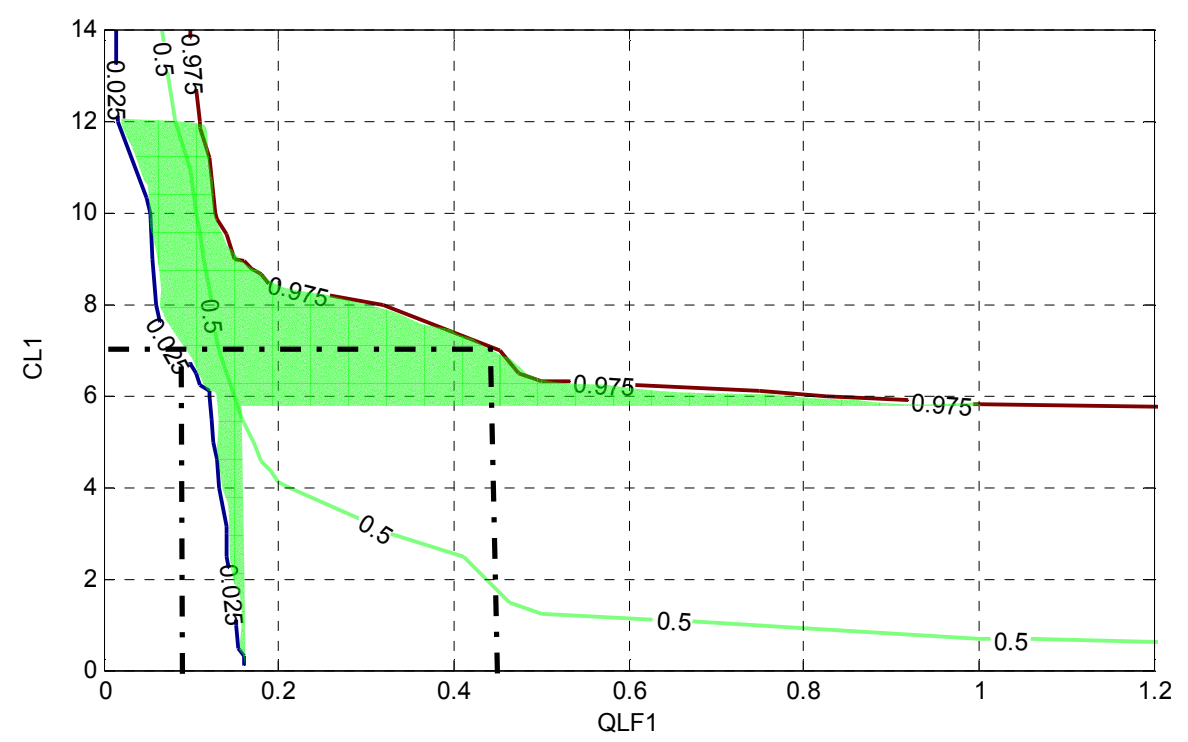

Fig. 13 Dependences between CL1 and QFL1; MRK17

For example, for a randomly chosen value of 7 for CL1 uncertainty, QLF1 uncertainty must be randomly selected between 0.07 and 0.45 (see on figure 13).

By drawing the intermediate percentile curves, it is also possible to obtain an "experimental" joint cumulated distribution function for the uncertainties of these two parameters.

\section{To Sum Up}

For these two Marviken tests, similar to the four Canon tests, the results (ranges of variation) are different: quasi independence for MRK24 and a rather strong dependence for MRK17. Finally, after analyzing MRK17 and MRK24, the uncertainty intervals are for CL1 $[0.05,12]$ and for QLF1 $[0.05,1]$. These ranges of variation are determined with the help of expert judgment. Indeed, according to the figure 13, we could select for CL1 a minimum value inferior to 0.05 and a maximum value superior to 12 . However, on our point of view the minimum value of an uncertainty interval cannot be less than 0.05 ; that is why we selected the previous intervals for CL1 and QLF1.

It will be noted that CL1 and QLF1 are involved in other separate effect tests: therefore for determining its uncertainty, we have to take into account all these experiments as TOBT1.

\section{Conclusion}

DIPE makes easy and automatic determination of the bounding interval for uncertainties. It is a precious help for the expert judgment. It reduces the user effect and 
gives an accurate estimation of the range of variation.

The parameter uncertainty PDF of each test is easily obtained but, due to lack of available experimental results, it is not possible to estimate an overall PDF.

The dependencies between parameter uncertainties can also be estimated but like in the previous case, the lack of experimental results is hindering.

It can also be noted that the mathematical assumptions may not always be verified. If the "Monotony" assumption is not verified, DIPE can nevertheless be used "part-wise" i.e. on time spans where monotony is verified.

Further development and application of DIPE is in progress to determine the statistical characteristics of all the input uncertain parameters of CATHARE2 V2.5_1. Its application for one or two parameters can be extended to more parameters.

\section{References}

(1)BESTION, D., \& al., Methodology, Status and Plans for Development and Assessment of CATHARE code, OECD/CSNI Workshop on Trans. Thermalhydr. and Neutr. Code Req., Annapolis (1996)

(2)FORGE, A., \& al., Review study on uncertainty methods for thermal-hydraulic computer codes, European Commission, Report EUR 15364 EN (1993)

(3)NEA/CSNI/R(97)35, Report on the uncertainty methods studies (1998)

(4)JOUCLA, J., PROBST, P., Rank Statistics and Bootstrap: a More Precise Evaluation of the $95^{\text {th }}$ Percentile in Nuclear Safety LB-LOCA Calculations, Proceedings of ICONE 14, Miami (2006)

(5)RIEGEL, B., MARECHAL, A., Expérience CANON - dépressurisation d'une capacité en double phase eau-vapeur, CEA, TT/547 (restricted) (1977)

(6)Marviken project team, The Marviken full Scale Critical Flow Tests - Description of the test facility, MXC-101 (restricted) (1979a)

(7)Marviken project team, The Marviken full Scale Critical Flow Tests - Results from Test 17, MXC-217 (restricted) (1979b)

(8)Marviken project team, The Marviken full Scale Critical Flow Tests - Results from Test 24, MXC-224 (restricted) (1979c) 